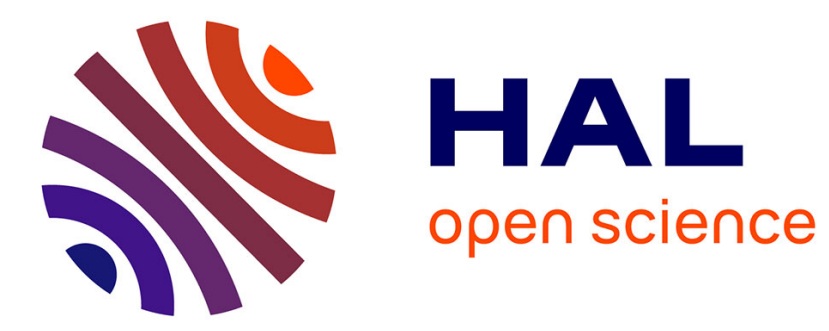

\title{
Pancyclic arcs and connectivity in tournaments
}

Frédéric Havet

\section{To cite this version:}

Frédéric Havet. Pancyclic arcs and connectivity in tournaments. RR-4378, INRIA. 2002. inria00072210

\section{HAL Id: inria-00072210 https://hal.inria.fr/inria-00072210}

Submitted on 23 May 2006

HAL is a multi-disciplinary open access archive for the deposit and dissemination of scientific research documents, whether they are published or not. The documents may come from teaching and research institutions in France or abroad, or from public or private research centers.
L'archive ouverte pluridisciplinaire HAL, est destinée au dépôt et à la diffusion de documents scientifiques de niveau recherche, publiés ou non, émanant des établissements d'enseignement et de recherche français ou étrangers, des laboratoires publics ou privés. 
INSTITUT NATIONAL DE RECHERCHE EN INFORMATIQUE ET EN AUTOMATIQUE

\section{Pancyclic arcs and connectivity in tournaments}

Frédéric Havet

$\mathbf{N}^{\circ} 4378$

Février 2002

THÈME 1 



\title{
Pancyclic arcs and connectivity in tournaments
}

\author{
Frédéric Havet \\ Thème 1 - Réseaux et systèmes \\ Projet Mascotte \\ Rapport de recherche $n^{\circ} 4378$ - Février 2002 - 19 pages
}

\begin{abstract}
A tournament is an orientation of the edges of a complete graph. An arc is pancyclic in a digraph $D$ if it is contained in a cycle of length $l$, for every $3 \leq l \leq|D|$. In [4], Moon showed that every strong tournament contains at least three pancyclics arcs and characterized the tournaments with exactly three pancyclic arcs. All these tournaments are not 2-strong. In this paper, we are interested in the minimum number $p_{k}(n)$ of pancyclic arcs in a $k$-strong tournament of order $n$. We conjecture that (for $k \geq 2$ ) there exists a constant $\alpha_{k}>0$ such that $p_{k}(n) \geq \alpha_{k} n$. After proving that every 2-strong tournament has a hamiltonian cycle containing at least five pancyclic arcs, we deduce that for $k \geq 2$, $p_{k}(n) \geq 2 k+3$. We then characterize the tournaments having exactly four pancyclic arcs and those having exactly five pancyclic arcs.
\end{abstract}

Key-words: pancyclic, tournament, strong connectivity, strongness 


\section{Arcs pancycliques et connexité dans les tournois}

Résumé : Un tournoi est une orientation des arêtes du graphe complet. Un arc est pancyclique dans un graphe orienté $D$ s'il est contenu dans un cycle de longueur $l$, pour toute longueur $3 \leq l \leq|D|$. Dans [4], Moon a montré que tout tournoi fortement connexe contenait au moins trois arcs pancycliques et il a caractérisé les tournois ayant exactement trois arcs pancycliques. Tous ces tournois ne sont pas 2-fortement connexes. Dans ce rapport, nous étudions le nombre minimum $p_{k}(n)$ d'arcs pancycliques dans un tournoi $k$ fortement connexe. Nous conjecturons que (pour $k \geq 2$ ), il existe une constante $\alpha_{k}$ telle que $f_{k}(n) \geq \alpha_{k} n$. Nous prouvons d'abord que tout tournoi 2-fortement connexe possède un circuit hamiltonien contenant au moins cinq arcs pancycliques. Nous en déduisons ensuite que pour $k \geq 2, p_{k}(n) \geq 2 k+3$. Enfin, nous caractérisons les tournois ayant exactement quatre arcs pancycliques et ceux ayant exactement cinq arcs pancycliques.

Mots-clés : pancyclique, tournoi, forte connexité 


\section{Introduction}

A tournament is an orientation of the arcs of a complete graph. Paths and cycles are always directed. A $l$-cycle is a cycle of length $l$.

An arc or a vertex is pancyclic in a digraph $D$ if, for every $3 \leq l \leq|D|$, it is contained in an $l$-cycle.

A tournament is strong (or strongly connected) if for any two vertices $x$ and $y$ there exists a path beginning in $x$ and terminating at $y$. A nonstrong tournament is said to be reducible. A tournament is $k$-strong, if $T-Y$ is strong for any set $Y$ of $k-1$ vertices. A tournament is $(=k)$-strong or exactly $k$-strong, if it is $k$-strong and not $(k+1)$-strong.

To contain a pancyclic arc or vertex, a tournament must contain a hamiltonian cycle. Therefore, it must be strong according to the well known theorem of Camion [2]: A tournament has a hamiltonian cycle if and only if it is strong. Moon [3] gave an alternative proof of Camion's theorem by proving that every vertex of a strong tournament is pancyclic.

Analogously, one may wonder whether there are pancyclic arcs in tournament and how many. Moon [4] showed that every strong tournament contains at least three pancyclic arcs. Actually, he proved a somewhat stronger result : indeed, instead of considering the number $p(T)$ of pancyclic arcs in the tournament $T$, he proved that $h(T)$ the maximum number of pancyclic arcs contained in some hamiltonian cycle of $T$ is at least 3 .

Theorem 1 (Moon, [4]) Let $T$ be a strong tournament with $n \geq 3$ vertices.

$$
h(T) \geq 3
$$

with equality holding only if $T \in \mathcal{P}_{3}$.

A tournament is in $\mathcal{P} 3$ if there is a vertex $v$ such that $T-v$ is the transitive tournament $T T\left[t_{1}, t_{2}, \ldots, t_{m}\right]\left(\left(t_{i}, t_{j}\right)\right.$ is an arc if and only if $\left.i<j\right)$, and an integers $1<i_{1} \leq m$ such that $v \rightarrow t_{j}$ if and only if $1 \leq j<i_{1}$.

Let $p_{k}(n)$ be minimum number $p_{k}(n)$ of pancyclic arcs in a $k$-strong tournament of order $n$ and let $h_{k}(n):=\min \{h(T) ; T k-$ strong of order $n\}$.

Because the tournaments of $\mathcal{P}_{3}$ are $(=1)$-strong, we have $p_{1}(n)=h_{1}(n)=3$ and if $k \geq 2$, $p_{k}(n) \geq h_{k}(n) \geq 4$. However we consider that this lower bound 4 is not tight.

In this paper, we show Section 3 sufficient conditions for an arc to be pancyclic in a tournament. Using these conditions, we give an easy alternative proof of Theorem 1. Moreover our method allow us to go further. In Section 4, we prove that for $k \geq 2, h_{k}(n) \geq 5$ and then deduce that $p_{k}(n) \geq 2 k+3$. Finally, we characterize the tournaments with exactly four pancyclic arcs and those with exactly five pancyclic arcs.

However, our lower bound for $h_{k}$ and $p_{k}$ seems to be still far from the exact value. We conjecture that for $k \geq 2, p_{k}(n)$ tends linearly to infinity :

Conjecture 1 For $k \geq 2$, there exists a constant $\alpha_{k}>0$ such that $p_{k}(n) \geq \alpha_{k} n$. 
We cannot expect to have more pancyclic arcs since there are $k$-strong tournaments having less than $2 k n$ pancyclic arcs.

Proposition $1 p_{k}(n) \leq 2 k n-2 k^{2}-k$

Proof. Let $T_{n}$ be the $k$-strong tournament obtained from the rotative tournament on $2 k+1$ vertices by blowing up a vertex with a transitive tournament $T T$ of order $n-2 k$. Every arc in $T T$ is not pancyclic in $T$ since it is contained in no 3 -cycle. Thus $(n-2 k)(n-2 k-1) / 2$ arcs are not pancyclic.

\section{Proposition 2}

$$
h_{k}(n) \leq 3 k
$$

Proof. If $n \leq 3 k$, we have trivially the answer. Suppose now that $n>3 k$. Consider the $k$-strong tournament $T_{n}$ obtained from two $T T_{k}, A$ and $B$, and one $T T_{n-2 k} C$ such that $A \rightarrow B \rightarrow C \rightarrow A$. It is easy to see that every arc contained in one of the three subtournaments $A, B$ and $C$ is not pancyclic because it is contained in no 3 -cycle. It follows that $h\left(T_{n}\right) \leq 3 k$.

The bound $3 k$ is not tight because of the 2 -strong tournaments of order $2 k+1 \leq n<3 k$. However, we think that if $n$ is large enough, the above example are extremal.

Conjecture 2 For $n$ sufficientlylarge, $h_{k}(n)=3 k$.

Alspach [1] showed that every arc of a regular tournament is pancyclic. This implies that $h_{k}(2 k+1)=2 k+1$. In order to avoid the exception of small order in Conjecture 2, it would make sense to first try to prove that $h_{k}(n) \geq 2 k+1$.

\section{Definition and preliminaries}

Let $T$ be a tournament. Let $x$ and $y$ be two vertices of $T$. We write $x \rightarrow y$ if $(x, y)$ is an arc of $T$. Likewise, let $X$ and $Y$ be two subdigraphs of $T$. We write $X \rightarrow Y$ if $x \rightarrow y$ for all pairs $(x, y) \in V(X) \times V(Y)$.

Let $A_{1}, A_{2}, \ldots, A_{k}$ be a family of subdigraphs of $T$. We denote by $T\left[A_{1}, A_{2}, \ldots, A_{k}\right]$ the subtournament induced by $T$ on the set of vertices $\bigcup_{1 \leq i \leq k} V\left(A_{i}\right)$ and by $T-\left[A_{1}, A_{2}, \ldots, A_{k}\right]$ the subtournament induced by $T$ on the set of vertices $V(T) \backslash \bigcup_{1 \leq i \leq k} V\left(A_{i}\right)$.

$A(X, Y)$ denotes the set of arc $(x, y)$ with $x \in X$ and $y \in Y . A^{+}(X)$ is the set of arcs outgoing from $X$, that is $A(X, T-X)$ and $A^{-}(X)$ is the set of arcs ingoing into $X$, that is $A(T-X, X)$. 
The dual of a digraph $D$ is the digraph $-D$ on the same set of vertices such that $x \rightarrow y$ is an arc of $-D$ if and only if $y \rightarrow x$ is an $\operatorname{arc}$ of $D$.

An $(x, y)$-path is a path that begins in $x$ and terminates at $y$.

One can easily show the following result (whose proof is left to the reader) that allows to extend an $(x, y)$ path to a longer one under condition.

Proposition 3 Let $P=\left(v_{1}, v_{2}, \ldots, v_{m}\right)$ be a path in a tournament $T$ and $x$ a vertex of $T-P$.

If there exist $1 \leq i<j \leq m$ such that $v_{i} \rightarrow x \rightarrow v_{j}$, then in $T$ there is a path with $m+1$ vertices starting in $v_{1}$ and ending in $v_{m}$.

A non-strong tournament $T$ is said to be reducible. It admits a reduction into two subtournaments $T_{1}$ and $T_{2}$ such that $V\left(T_{1}\right) \cup V\left(T_{2}\right)=V(T)$ and $T_{1} \rightarrow T_{2}$; in this case, we write $T=T_{1} \rightarrow T_{2}$.

A (strong) component of $T$ is a strong subtournament of $T$ which is maximal by inclusion. Let $T_{1}, T_{2}, \ldots, T_{m}$ be the components of $T$. Then $\left(V\left(T_{1}\right), V\left(T_{2}\right), \ldots, V\left(T_{m}\right)\right)$ is a partition of $V(T)$ and without loss of generality, we may suppose that $T_{i} \rightarrow T_{j}$ whenever $i<j$. In this case we say that $T_{1} \rightarrow T_{2} \rightarrow \cdots \rightarrow T_{m}$ is the decomposition of $T$. The component $T_{1}$ (resp. $T_{m}$ ) is called the outsection (resp. insection) of $T$, denoted by out $(T)$ (resp. $\operatorname{In}(T)$ ) and its vertices are called the outgenerators of $T$ (resp. ingenerators of $T$.

Proposition 4 In a tournament, a vertex is the beginning of a hamiltonian path if and only if it is an outgenerator.

Proof. Let $T$ be a tournament. Then $T=\operatorname{Out}(T) \rightarrow T-\operatorname{Out}(T)$ (with $T-\operatorname{Out}(T)$ empty when $T$ is strong). $T[$ Out $(T)]$ is strong, and thus by Camion's theorem admits a hamiltonian cycle $C$. Then every outgenerator $v$ is the origin of a hamiltonian path $Q$ of $T[O u t(T)]$. And $T-\operatorname{Out}(T)$ has a hamiltonian path $P$. So $(P, Q)$ is hamiltonian path of $T$ with origin $v$.

Proposition 5 Let $T$ be a reducible tournament of order $n$. Let $u$ be an ingenerator and $t$ an outgenerator of $T$. For any $1 \leq l \leq n-1$, there is a $(t, u)$-path of length $l$.

Proof. Let $T_{1} \rightarrow T_{2}$ be a reduction of $T$ and for $i=1,2$, let $n_{i}$ be the order of $T_{i}$. Clearly, $t$ is an ingenerator of $T_{2}$ and $u$ an outgenerator of $T_{1}$. Therefore, by Proposition $4, t$ is the end of a hamiltonian path $\left(t_{n_{2}-1}, t_{n_{2}-2}, \ldots, t_{1}, t\right)$ of $T_{2}$ and $u$ is the origin of a hamiltonian path $\left(u, u_{1}, \ldots, u_{n_{1}-2}, u_{n_{1}-1}\right)$ of $T_{1}$. Now, for any $1 \leq l \leq n-1$, pick $0 \leq l_{1} \leq n_{1}-1$ and $0 \leq l_{2} \leq n_{2}-1$ such that $l_{1}+l_{2}=l-1$. Then $\left(u, u_{1}, \ldots, u_{l_{1}}, t_{l_{2}}, t_{l_{2}-1}, \ldots, t\right)$ is a path of length $l$.

A reductor of a tournament is a smallest subtournament $X$ such that $T-X$ is reducible. If $T$ is $(=k)$-strong then a reductor has $k$ vertices.

Proposition 6 Let $X$ be a reductor of a tournament $T$. Every element of $X$ dominates an outgenerator of $T-X$ and is dominated by an ingenerator of $T-X$.

$\mathrm{RR} \mathrm{n}^{\circ} 4378$ 
Proof. Let $x$ be an element of $X$. Let $Y$ be the set of vertices that are not outgenerator of $T-X$. If $\operatorname{Out}(T-X) \rightarrow x$, then $T-[X \backslash x]$ is reducible with reduction $O u t(T-X) \rightarrow T[Y, x]$. This contradicts that $X$ is a reductor.

Analogously, we prove that $x$ is dominated by an ingenerator of $T-X$.

Proposition 7 Let $x$ and $y$ be two vertices of a a reductor $X$ of a 2-tournament $T$. If in $(T-X) \geq 3$, there are two distinct vertices $z_{x}$ and $z_{y}$ of $\operatorname{In}(T-X)$ such that $z_{x} \rightarrow x$ and $z_{y} \rightarrow y$.

Proof. Suppose that two such vertices do not exist, then by Proposition 6 , there is a vertex $u \in \operatorname{In}(T-X)$ such that $u \rightarrow\{x, y\}$ and $\operatorname{In}(T-X) \backslash u \leftarrow\{x, y\}$. Then $X \backslash\{x, y\} \cup\{u\}$ is a reductor of $T$, which is a contradiction.

\section{Lower bounds for $p(T)$ and $h(T)$}

\subsection{Sufficient conditions for an arc to be pancyclic}

Lemma 1 Let $X$ be a subtournament such that $T-X$ is reducible and every vertex of $X$ dominates an outgenerator of $T-X$. Let $v$ be an outgenerator of $X$ and $u$ an ingenerator of $T-X$. If $u \rightarrow v$, then the arc $(u, v)$ is pancyclic.

Proof. By Proposition $4, v=v_{0}$ is the origin of a hamiltonian path $\left(v_{0}, v_{1}, \ldots, v_{k-2}, v_{k-1}\right)$ of $T[X]$. For $0 \leq i \leq k-1$, let $t_{i}$ be an outgenerator of $T-X$ dominated by $v_{i}$. For $3 \leq l \leq n$, take $0 \leq k^{\prime} \leq k-1$ and $1 \leq l \leq n-k-1$ such that $k^{\prime}+l+2=l$. Then by Proposition 5 , in $T-X$, there is $\left(t_{k^{\prime}}, u\right)$ path $Q$ of length $l$. Thus $\left(v_{0}, v_{1}, \ldots, v_{k^{\prime}}, Q, v_{0}\right)$ is a cycle of length $l$ going through $(u, v)=\left(u, v_{0}\right)$.

Corollary 1 Let $X$ be the reductor of a tournament $T$. Let $v$ be an outgenerator of $X$ and $u$ an ingenerator of $T-X$. If $u \rightarrow v$, then the arc $(u, v)$ is pancyclic.

Corollary 2 Let $T$ be a strong tournament.

$$
p(T) \geq h(T) \geq 2
$$

Proof. Let $X$ be a reductor of $T$. Let $P$ be a hamiltonian path of $X$ with beginning $v$ and end $w$. Clearly, $v$ is an outgenerator of $X$ and $w$ is an ingenerator of $X$. Then by Corollary 1 (and its dual), $(u, v)$ and $(w, t)$ are pancyclic.

And by Proposition 5 , there is a $(t, u)$-path $Q$ that is hamiltonian in $T-X$, thus $(P, Q, v)$ is a hamiltonian cycle containing $(u, v)$ and $(w, t)$.

Let $T$ be a strong tournament $T$ with reductor $X$. Let $X_{1} \rightarrow X_{2} \rightarrow \cdots \rightarrow X_{l}$ be a decompositon of $X$ and $T_{1} \rightarrow T_{2} \rightarrow \cdots \rightarrow T_{m}$ be a decomposition of $T-X$.

In the remaining of this section, we examine the number of pancyclic arcs in the different parts of $T$.

INRIA 
Lemma 2 For $1 \leq i \leq l$, if an arc is pancyclic in $X_{i}$, then it is also pancyclic in $T$.

Proof. Let $e$ be a pancyclic arc in one of the $X_{i}$. Then $e$ is contained in a 3-cycle. It is also contained in a hamiltonian cycle and then a hamiltonian path of $X_{i}$. This hamiltonian path may be extended to a hamiltonian path $\left(v_{0}, v_{1}, \ldots, v_{k-1}\right)$ of $X$ using hamiltonian paths of the $X_{i^{\prime}}$, for $i^{\prime} \neq i$. Let $j$ be the index such that $e=\left(v_{j}, v_{j+1}\right)$. Let $4 \leq l \leq n$. Choose $0 \leq l_{1} \leq j, 0 \leq l_{2} \leq k-j-2$ and $1 \leq l_{3} \leq n-k-1$ such that $l_{1}+l_{2}+l_{3}+3=l$. By Proposition 6 , there is an ingenerator $u$ of $T-X$ dominating $v_{j-l_{1}}$ and an outgenerator $t$ of $T-X$ dominated by $v_{j+1+l_{2}}$. Then by Proposition 5 , in $T-X$ there is a $(t, u)$-path of length $l_{3}$. Hence $\left(v_{j-l_{1}}, v_{j-l_{1}+1}, \ldots, v_{j+1+l_{2}}, P, v_{j-l_{1}}\right)$ is an $l$-cycle containing $e$.

Lemma 3 Suppose that $T-X$ is the transitive tournament $T T\left(t_{1}, t_{2}, \ldots, t_{m}\right)$.

For every $1 \leq i \leq m-1$, the arc $\left(t_{i}, t_{i+1}\right)$ is pancyclic if and only if it is contained in $a$ 3-cycle.

Proof. By Proposition $6, X \rightarrow t_{1}$ and $t_{m} \rightarrow X$. Let $\left(v_{0}, v_{1}, \ldots, v_{k-2}, v_{k-1}\right)$ be a hamiltonian path of $X$.

Suppose first that $i=1$. Let $4 \leq l \leq n$. Take $0 \leq k^{\prime}<k$, and $1 \leq l^{\prime}<m-2$ such that $k^{\prime}+l^{\prime}+3=l$. By Proposition 5 , there is a $\left(t_{2}, t_{m}\right)$-path $P$ of length $l^{\prime}$ in $T\left[t_{2}, \ldots, t_{m}\right]$. Then $\left(t_{1}, P, v_{0}, v_{1}, \ldots, v_{k^{\prime}}, t_{1}\right)$ is a cycle of length $l$. Thus if $\left(t_{1}, t_{2}\right)$ is contained in a 3 -cycle then it is pancyclic.

By duality, we have the result if $i=m-1$.

Suppose now that $1<i<m-1$. Let $v$ be the vertex such that $\left(t_{i}, t_{i+1}, v, t_{i}\right)$ is a 3-cycle. Necessarily, $v$ is in $X$. Then $\left(t_{i}, t_{i+1}, v, t_{1}, t_{i}\right)$ is a 4 -cycle. Let $5 \leq l \leq n$. The $\operatorname{arc}\left(t_{i}, t_{i+1}\right)$ is contained in a cycle of length $l$. Indeed, take $0 \leq k^{\prime}<k, 0<l_{1}<i$ and $0 \leq l_{2}<m-i$ such that $k^{\prime}+l_{1}+l_{2}+3=l$. By Proposition 5 , there is a $\left(t_{1}, t_{i}\right)$-path $P_{1}$ of length $l_{1}$ in $T\left[t_{1}, \ldots, t_{i}\right]$, and a $\left(t_{i+1}, t_{m}\right)$-path $P_{2}$ of length $l_{2}$ in $T\left[t_{i+1}, \ldots, t_{m}\right]$. Then $\left(t_{i}, P_{2}, v_{0}, v_{1}, \ldots, v_{k^{\prime}}, P_{1}\right)$ is an $l$-cycle containing $\left(t_{i}, t_{i+1}\right)$. Thus $\left(t_{i}, t_{i+1}\right)$ is pancyclic.

Lemma 4 For $1<i<m$, if $\left|T_{i}\right| \geq 4$, then every $T_{i}$-pancyclic arc is pancyclic in $T$.

Proof. Let $\left(v_{0}, v_{1}, \ldots, v_{k-2}, v_{k-1}\right)$ be a hamiltonian path of $X$.

Let $\left(a_{1}, a_{2}\right)$ be a pancyclic arc in $T_{i}$. It is contained in a hamiltonian cycle of $T_{i}, C_{i}=$ $\left(a_{1}, a_{2}, \ldots, a_{n_{i}}, a_{1}\right)$.

Let us prove that $\left(a_{1}, a_{2}\right)$ is contained in a cycle of length $l$ for all $5 \leq l \leq n$. Let $S_{1}=T\left[T_{1}, T_{2}, \ldots, T_{i-1}, a_{1}\right]$ and $S_{2}=T-\left[X, S_{1}\right]$. Clearly, $a_{1}$ is an ingenerator of $S_{1}$ and $a_{2}$ an outgenerator of $S_{2}$. There exists three integers $1 \leq l_{1} \leq\left|S_{1}\right|-1,1 \leq l_{1} \leq\left|S_{2}\right|-1$ and $0 \leq k^{\prime} \leq k-1$ such that $l_{1}+l_{2}+k^{\prime}=l-3$. By Proposition 6 , there is an ingenerator $u_{0}$ of $\bar{T}-\bar{X}$ and then also of $S_{2}$ which dominates $v_{0}$ and an outgenerator $t_{k^{\prime}}$ of $S_{1}$ which is dominated by $v_{k^{\prime}}$. By Proposition 5 , there is a $\left(t_{k^{\prime}}, a_{1}\right)$-path $P_{1}$ of length $l_{1}$ in $S_{1}$ and a $\left(a_{2}, u_{0}\right)$-path $P_{2}$ of length $l_{2}$ in $S_{2}$. Then $\left(P_{1}, P_{2}, v_{0}, v_{1}, \ldots v_{k^{\prime}}, t_{k^{\prime}}\right)$ is the desired $l$-cycle.

And because it is pancyclic in $T_{i},\left(a_{1}, a_{2}\right)$ is contained in an $l$-cycle, for $3 \leq l \leq n_{i}$.

Lemma 5 For any $1<i<m$, if $T_{i}$ is a 3-cycle, then two arcs of $T_{i}$ are pancyclic in $T$. 
Proof. Let $\left(v_{0}, v_{1}, \ldots, v_{k-2}, v_{k-1}\right)$ be a hamiltonian path of $X$. The component $T_{i}$ is the 3-cycle $\left(a_{1}, a_{2}, a_{3}, a_{1}\right)$.

In the same way as in the proof of Lemma 4 the arc $\left(a_{1}, a_{2}\right)$, is contained in cycle of length $l$ for all $5 \leq l \leq n$. Analogously, $\left(a_{2}, a_{3}\right)$ and $\left(a_{3}, a_{1}\right)$ are contained in cycle of length $l$ for all $5 \leq l \leq n$.

Hence, it suffice to prove that two arcs of $T_{i}$ is contained in a 4-cycle. Without loss of generality, we may assume that $\left\{a_{1}, a_{2}\right\} \leftarrow v_{0}$ or $\left\{a_{1}, a_{2}\right\} \rightarrow v_{0}$. If $\left\{a_{1}, a_{2}\right\} \leftarrow v_{0}$, then $\left(a_{1}, a_{2}, u_{0}, v_{0}, a_{1}\right)$ and $\left(a_{2}, a_{3}, u_{0}, v_{0}, a_{2}\right)$ are 4-cycles. If $\left\{a_{1}, a_{2}\right\} \rightarrow v_{0}$, then $\left(a_{3}, a_{1}, v_{0}, t_{0}, a_{3}\right)$ and $\left(a_{1}, a_{2}, v_{0}, t_{0}, a_{1}\right)$ are 4-cycles.

Lemma 6 Let $C=\left(a_{1}, a_{2}, \ldots, a_{n_{1}}, a_{1}\right)$ be a hamiltonian cycle of $T_{1}$ such that $a_{1}$ is dominated by an ingenerator of $X$.

If $\left(a_{j}, a_{j+1}\right)$ is pancyclic in $T_{1}$ and $1 \leq j \leq n_{1}-2$ then $\left(a_{j}, a_{j+1}\right)$ is pancyclic in $T$.

Proof. By $T_{1}$-pancyclicity, $\left(a_{j}, a_{j+1}\right)$ is contained in a cycle of length $l$ for $3 \leq l \leq n_{1}$.

Let now $l$ be an integer of $\left[n_{1}+1, n\right]$.

Let $v_{k-1}$ be an ingenerator of $X$ dominating $a_{1}$. By Proposition 4, there exists a hamiltonian path $\left(v_{0}, v_{1}, \ldots, v_{k-2}, v_{k-1}\right)$ of $T[X]$. By Proposition 6 , for every $0 \leq i \leq k-1$, there is an ingenerator $u_{i}$ of $T-X$ dominating $v_{i}$.

Let $1 \leq l_{1} \leq n-k-j-1$ and $0 \leq k^{\prime} \leq k-1$ such that $k^{\prime}+l_{1}=l-j-2$. Obviously, $a_{j+1}$ is an outgenerator of $T^{\prime}=T-\left[X, a_{1}, a_{2}, \ldots, a_{j}\right]$. And $u_{k-1-k^{\prime}}$ is an ingenerator of $T^{\prime}$. Thus, by Proposition 5 there is an $\left(a_{j+1}, u_{k-1-k^{\prime}}\right)$ path $P$ of length $l_{1}$. Hence $\left(P, v_{k-1-k^{\prime}}, v_{k-1-k^{\prime}}, \ldots, v_{k-1}, a_{1}, a_{2}, \ldots, a_{j+1}\right)$ is a cycle of length $l$.

Lemma 7 There are at least $h\left(T_{1}\right)-1$ pancyclic arcs in $T_{1}$.

Proof. Let $C=\left(a_{1}, a_{2}, \ldots, a_{n_{1}}, a_{1}\right)$ be a hamiltonian cycle of $T_{1}$ containing $h\left(T_{1}\right) T_{1^{-}}$ pancyclic arcs. Without loss of generality, we may suppose that $a_{1}$ is dominated by an ingenerator $v$ of $X$.

Since $j_{1} \leq n_{1}-2$, by Lemma 6 , every pancyclic arc in $P=\left(a_{1}, a_{2}, \ldots, a_{n_{1}-1}\right)$ is also pancyclic in $T$. If $P$ contains $h\left(T_{1}\right)-1 T_{1}$-pancyclic arcs, we have the result.

Hence we may assume that $\left(a_{n_{1}-1}, a_{n_{1}}\right)$ and $\left(a_{n_{1}}, a_{1}\right)$ are pancyclic in $T_{1}$.

If $v \rightarrow a_{n_{1}}$, again by Lemma 6 , the arc $\left(a_{n_{1}}, a_{1}\right)$ is pancyclic in $T$.

If $v \leftarrow a_{n_{1}}$ then let us prove that $e_{2}=\left(a_{n_{1}-1}, a_{n_{1}}\right)$ is pancyclic in $T$. By pancyclicity in $T_{1}, e_{2}$ is contained in a cycle of length $l$ for $3 \leq l \leq n_{1}$. And $\left(a_{1}, a_{2}, \ldots, a_{n_{1}}, v, a_{1}\right)$ is an $\left(n_{1}+1\right)$-cycle containing $e_{2}$.

Let now $l$ be an integer of $\left[n_{1}+2, n\right]$.

By Proposition 4, there is a hamiltonian path such that $\left(v_{0}, v_{1}, \ldots, v_{k-2}, v_{k-1}\right)$ be a hamiltonian path of $X$. By Proposition 6 , for every $0 \leq i \leq k-1$, there is an ingenerator $u_{i}$ of $T-X$ dominating $v_{i}$.

Let $1 \leq l_{1} \leq n-k-n_{1}-1$ and $0 \leq k^{\prime} \leq k-1$ such that $k^{\prime}+l_{1}=l-n_{1}-1$. Obviously, $a_{n_{1}}$ is an outgenerator of $T^{\prime}=T-\left[X, O u t(X) \backslash a_{n_{1}}\right]$. And $u_{k-1-k^{\prime}}$ is an ingenerator of $T^{\prime}$. Thus, by Proposition 5 there is a path $P$ of length $l_{1}$ with start $a_{n_{1}}$ and end $u_{k-1-k^{\prime}}$. Hence $\left(P, v_{k-1-k^{\prime}}, v_{k-1-k^{\prime}}, \ldots, v_{k-1}, a_{1}, a_{2}, \ldots, a_{n_{1}}\right)$ is a cycle of length $l$. 


\subsection{The lower bounds}

Using the above lemmas, we derive lower bounds for $p(T)$ and $h(T)$.

Definition 1 Let $k$ be an integer and $T$ a tournament. $\epsilon(k, T)=1$ if $|T| \geq k$ and 0 otherwise.

Theorem 2 If $T-X$ is transitive then

$$
p(T) \geq|A(\operatorname{In}(T-X) ; O u t(X))|+|A(\operatorname{In}(X) ; O u t(T-X))|+\sum_{j=1}^{l} p\left(X_{j}\right)+1
$$

Otherwise

$$
\begin{aligned}
p(T) \geq \quad & |A(\operatorname{In}(T-X) ; \operatorname{Out}(X))|+|A(\operatorname{In}(X) ; \operatorname{Out}(T-X))|+\sum_{j=1}^{l} p\left(X_{j}\right) \\
& +\sum_{i=2}^{m-1}\left\{2 \epsilon\left(3, T_{i}\right)+\left(p\left(T_{i}\right)-2\right) \epsilon\left(4, T_{i}\right)\right\} \\
& +\epsilon\left(3, T_{1}\right)\left(h\left(T_{1}\right)-1\right)+\epsilon\left(3, T_{m}\right)\left(h\left(T_{m}\right)-1\right)
\end{aligned}
$$

Proof. Since every outgenerator of $T-X$ is dominated by an ingenerator of $T-X$, according to Corrolary 1 , every arc of $A(\operatorname{In}(T-X) ; O u t(X))$ is pancyclic. By duality, every arc of $A(\operatorname{In}(X) ; \operatorname{Out}(T-X))$ is pancyclic. According to Lemma 2, there are at least $\sum_{j=1}^{l} p\left(X_{j}\right)$ $T$-pancyclic arcs in $X$.

Suppose now that $T-X$ is a transitive tournment. Then since $v_{0} \rightarrow t_{1}$ and $v_{0} \rightarrow t_{m}$ then there is an index $i$ such that $\left(t_{i}, t_{i+1}, v_{0}, t_{i}\right)$ is a 3 -cycle. So by Lemma 3 , this arc is pancyclic. So we obtain Equation 1.

To obtain Equation 2, let us now count the number of pancyclic arcs contained in each $T_{i}$ such that $\left|T_{i}\right| \geq 3$. If $i=1$ or $i=m$, by Lemma 7 (or its dual), $h\left(T_{i}\right)-1$ arc are $T$-pancyclic.

If $1<i<m$, then, if $T_{i}$ is a 3 -cycle then by Lemma $5,2 \operatorname{arcs}$ of $T_{i}$ are pancyclic in $T$; and if $\left|T_{i}\right| \geq 4$, by Lemma 4 , each $T_{i}$-pancyclic arc is pancyclic in $T$.

Theorem 3 If $T-X$ is transitive then

$$
h(T) \geq 3+\sum_{j=1}^{l} \epsilon\left(3, X_{j}\right) \cdot \min \left\{h\left(X_{j}\right) ;\left|X_{j}\right|-1\right\}
$$

otherwise

$$
\begin{aligned}
h(T) \geq & 2+\sum_{j=1}^{l} \epsilon\left(3, X_{j}\right) \cdot \min \left\{h\left(X_{j}\right) ;\left|X_{j}\right|-1\right\}+\sum_{i=2}^{m-1} \epsilon\left(3, T_{i}\right) \cdot \min \left\{h\left(T_{i}\right) ;\left|T_{i}\right|-1\right\} \\
& +\epsilon\left(3, T_{1}\right)\left(h\left(T_{1}\right)-1\right)+\epsilon\left(3, T_{m}\right)\left(h\left(T_{m}\right)-1\right)
\end{aligned}
$$

$\mathrm{RR} \mathrm{n}^{\circ} 4378$ 
Proof. For $1 \leq j \leq l$, let $P_{j}$ be a hamiltonian path of $X_{j}$ defined as follows :

- If $X_{j}$ is reduced to a single vertex $x_{j}$ then $P_{j}=\left(x_{j}\right)$.

- if $\left|X_{j}\right| \leq 3$, let $P_{j}$ is obtained from a hamiltonian cycle of $X_{j}$ containing $h\left(X_{j}\right) X_{j^{-}}$ pancyclic arcs by removing a non $X_{j}$-pancyclic arc if $h\left(X_{j}\right)<\left|X_{j}\right|$ or any arc if $h\left(X_{j}\right)=\left|X_{j}\right|$.

By Lemma 2 , each $P_{j}$ contains $\epsilon\left(3, X_{j}\right) . \min \left\{h\left(X_{j}\right) ;\left|X_{j}\right|-1\right\} T$-pancyclic arcs. Let $v$ be the beginning of $P_{1}$ and $w$ the end of $P_{l}$.

Suppose first that $T-X$ is the transitive tournament $T T\left(t_{1}, t_{2}, \ldots, t_{m}\right)$. By Lemma 3 , there exists $i$ such that $\left(t_{i}, t_{i+1}\right)$ is pancyclic and by Corollary $1,\left(w, t_{1}\right)$ and $\left(t_{m}, v\right)$ are pancyclic. Thus, the hamiltonian cycle $\left(\left(t_{1}, t_{2}, \ldots, t_{m}, P_{1}, P_{2}, \ldots, P_{l}, t_{1}\right)\right.$ contains $3+\sum_{j=1}^{l} \epsilon\left(3, X_{j}\right) \cdot \min \left\{h\left(X_{j}\right) ;\left|X_{j}\right|-\right.$ $1\}$.

Suppose now that $T-X$ is not transitive.

For $1<i<m$, let $Q_{i}$ be a hamiltonian path of $T_{i}$ defined as follows :

- If $T_{i}$ is reduced to a single vertex $t_{i}$ then $Q_{i}=\left(t_{i}\right)$.

- If $\left|T_{i}\right|=3$, then $Q_{i}$ is a path formed by two $\operatorname{arcs}$ of $T_{i}$ that are pancyclic in $T$. (Such arcs exists according to Lemma 5.)

- If $\left|T_{i}\right| \leq 4$, let $Q_{i}$ is obtained from a hamiltonian cycle of $T_{i}$ containing $h\left(T_{i}\right) T_{i^{-}}$ pancyclic arcs by removing a non $T_{i}$-pancyclic arc if $h\left(T_{i}\right)<\left|T_{i}\right|$ or any $\operatorname{arc}$ if $h\left(T_{i}\right)=$ $\left|T_{i}\right|$.

By Lemma 4, each $Q_{i}$ contains $\epsilon\left(3, T_{i}\right) . \min \left\{h\left(T_{i}\right) ;\left|T_{i}\right|-1\right\} T$-pancyclic arcs.

Let $C_{1}=\left(a_{1}, a_{2}, \ldots, a_{n_{1}}, a_{1}\right)$ be a hamiltonian path of $T_{1}$ containing $h\left(T_{1}\right) T_{1}$-pancyclic arcs. Then set $Q_{1}:=\left(\left(a_{1}, a_{2}, \ldots, a_{n_{1}}\right)\right.$. Analogously define $Q_{m}$. By (the proof of) Lemma 6 $Q_{1}$ (resp. $\left.Q_{m}\right)$ contains at least $h\left(T_{1}\right)-1$ (resp. $h\left(T_{m}\right)-1$ ) pancyclic $\operatorname{arcs}$ in $T$.

Then the hamiltonian cycle $\left(w, Q_{1}, Q_{2}, \ldots, t_{m}, P_{1}, P_{2}, \ldots, P_{l}\right)$ gives Equation 4.

\subsection{Tournaments with $h(T)=3$}

From the lower bounds, one can easily derive Theorem 1 :

Proof of Theorem 1 Let us prove the result by induction on the order $n$ of $T$. If $n=3$, it is obviously true.

Suppose now that it is true for strong tournaments of order less than $n$. Let $X$ be a reductor of $T$ and $T_{1} \rightarrow T_{2} \rightarrow \cdots \rightarrow T_{m}$ be a decomposition of $T-X$.

If $T-X$ is transitive, Equation 3 yields the result and if it is not then Equation 4 gives $h(T) \geq 4$.

Suppose now $T$ is a tournament such that $h(T)=3$. Let $X$ be a reductor of $T$. By Equation 4 then $X$ and $T-X$ are a transitive tournaments. Set $X=T T\left[v_{0}, v_{1}, \ldots, v_{k-1}\right]$ and $T-X=T T\left[t_{1}, t_{2}, \ldots, t_{n-k}\right]$. By Corollary $1,\left(t_{n-k}, v_{0}\right)$ and $\left(v_{k-1}, t_{1}\right)$ are pancyclic. Therefore there is at most one pancyclic arc on the hamiltonian path of $T-X$. Thus by

INRIA 
Lemma 3, there is an index $i$ such that $X \rightarrow t_{j}$ if and only if $j \leq i$. And $\left(t_{i}, t_{i+1}\right)$ is pancyclic.

Suppose that $T$ is 2 -strong. Then $T-t_{1}$ and $T-t_{n-k}$ are strong, so $2 \leq i \leq$ $n-k-2$. By Lemma $1,\left(t_{n-k-1}, v_{0}\right)$ is pancyclic in $T-t_{n-k}$ and $\left(t_{n-k}, v_{1}\right)$ is pancyclic in $T-v_{0}$. Thus two arcs are $T$-pancyclic because they are contained in the $n$ cycle $C_{3}=\left(t_{n-k-1}, v_{0}, v_{k-1}, t_{2}, t_{3}, \ldots, t_{n-k-2}, t_{n-k}, v_{1}, v_{2}, \ldots, v_{k-2}, t_{1}, t_{n-k-1}\right)$ if $k \geq 3$ or $C_{2}=\left(t_{n-3}, v_{0}, t_{1}, t_{n-2}, v_{1}, t_{2}, t_{3}, \ldots, t_{n-3}\right)$ if $k=2$. Analogously, $\left(v_{k-1}, t_{2}\right)$ and $\left(v_{k-2}, t_{1}\right)$ are also $T$-pancyclic. Hence the cycle $C_{2}$ or $C_{3}$ contains four pancyclic arcs. This is a contradiction.

Thus $T$ is $(=1)-$ strong. Then by Lemma 3 it is in $\mathcal{P}_{3}$.

Note that in the proof of Theorem 1, we also show the following :

Proposition 8 Let $X$ be a reductor of a strong tournament. There is at least one $T$ pancyclic arc in $T-X$.

\section{Number of pancyclic arcs in $k$-strong tournaments}

Theorem 4 (Yao, Guo and Zhang, [5]) Every tournament contains a vertex $x$ such that every outgoing arc is pancyclic.

From this result, we derive lower bounds on the number of pancyclic arcs in a strong tournament :

\section{Lemma 8}

$$
\begin{aligned}
& p(T) \geq h(T)+\delta^{+}-1 \\
& p(T) \geq h(T)+\delta^{+}+\delta^{-}-3
\end{aligned}
$$

Proof. By Theorem 4 and its dual, there is a vertex $x$ such that every outgoing arc is pancyclic and a vertex $y$ such that every ingoing arc is pancyclic. Obviously, $\mid A^{+}(x) \cap$ $A^{-}(y) \mid \leq 1$. There are $h(T)$ pancyclic arcs on a hamiltonian cycle. At most one of them is in $A^{+}(X)$ and at most two of them are in $A^{+}(x) \cup A^{-}(y)$. Hence, $p(T) \geq h(T)+d^{+}(x)-1 \geq$ $h(T)+\delta^{+}-1$, and

$$
\begin{aligned}
p(T) & \geq d^{+}(x)+d^{-}(y)-\left|A^{+}(x) \cap A^{-}(y)\right|+h(T)-2 \\
& \geq d^{+}(x)+d^{-}(y)+h(T)-3
\end{aligned}
$$

Lemma 9 Let $X$ be the reductor of a 2-strong tournament. Suppose that $X$ is the transitive tournament $T T\left(v_{0}, v_{1}, \ldots, v_{k-1}\right)$.

For every $0 \leq j \leq k-2$, the arc $\left(v_{j}, v_{j+1}\right)$ is pancyclic if and only if it is contained in a 3-cycle.

$\mathrm{RR} \mathrm{n}^{\circ} 4378$ 
Proof. Let $0 \leq j \leq k-2$ and $4 \leq l \leq n$. Let us prove that $\left(v_{j}, v_{j+1}\right)$ is contained in an $l$-cycle. There exist $0 \leq l_{1} \leq j, 0 \leq l_{2} \leq k-j-2$ and $1 \leq l_{3} \leq n-k$ such that $l_{1}+l 2+l_{3}+3=l$. By Propositon 6 , there is an ingenerator $u$ of $T-X$ dominating $v_{j-l_{1}}$ and an outegenerator $t$ of $T-X$ dominated by $v_{j+1+l_{2}}$. And by Proposition 5 , in $T-X$, there is an $(t, u)$-path $P$ of length $l_{3}$. Therefore, $\left(P, v_{j-l_{1}}, v_{j-l_{1}+1}, \ldots, v_{j+1+l_{2}}, t\right)$ is the desired $l$-cycle.

Theorem 5 For every a 2-strong tournament $T, h(T) \geq 5$.

Proof. Let $T$ be a 2-strong tournament such that $h(T)=4$. Let $X$ be a reductor of $T$ and $T_{1} \rightarrow T_{2} \rightarrow \cdots \rightarrow T_{m}$ be a decomposition of $T-X$. By Equations 3 and 4 , we may assume that $X$ is a transitive tournament, say $T T\left(v_{0}, v_{1}, \ldots, v_{k-1}\right)$, and that at most one of the $T_{i}$ is not reduced to a single vertex $t_{i}$.

I) Suppose that for some $2 \leq i \leq m-1, T_{i}$ is not reduced to a single vertex.

Then by Equation 4, we may assume that $T_{i}$ is a 3 -cycle $(a, b, c, a)$. By Lemma 10, for $1 \leq j \leq i-1, X \rightarrow t_{j}$ and for $i+1 \leq j \leq m, X \leftarrow t_{j}$. Without loss of generality, we may assume that both $a$ and $b$ dominate a vertex in $X$. Then by Lemma 10, $\left(t_{i-1}, a\right)$ and $\left(t_{i-1}, b\right)$ are pancyclic and by (the proof of ) Lemma 5 , the arcs $(a, b)$ and $(c, a)$ are pancyclic. If $c$ dominates a vertex in $X$ then $(b, c)$ is pancyclic, and if $c$ is dominated a vertex in $X$ then $\left(c, t_{i+1}\right)$ is pancyclic. In any case, the hamiltonian cycle $\left(t_{1}, t_{2}, \ldots, t_{i-1}, a, b, c, t_{i+1}, \ldots, t_{m}, v_{0}, v_{1}, \ldots, v_{k-1}, t_{1}\right)$ contains five pancyclic arcs.

II) Suppose that $\left|T_{1}\right| \geq 3$.

By Equation 4, we may assume that $h\left(T_{1}\right)=3$. So $T_{1} \in \mathcal{P}_{3}$ according to Theorem 1 . Let $w$ be the reductor of $T_{1}$ such that $T_{1}-w$ is the transitive tournament.

If for some $2 \leq j<m, t_{j}$ is dominated by a vertex of $X$, then by Lemma 10 (dual), there is a pancyclic arc in the path $\left(t_{j}, t_{j+1}, \ldots, t_{m}\right)$. Let $P$ be a hamiltonian path of $T_{1}$ beginning at an outneighbour of $V_{k-1}$. The hamiltonian cycle $C=\left(v_{k-1}, P, t_{2}, \ldots, t_{i-1}, t_{i}, t_{i+1}, \ldots, t_{m}, v_{0}, v_{1}, \ldots, v_{k-1}\right)$ contains five pancyclic arcs. Hence we may assume that $X \leftarrow t_{j}$, for $2 \leq j \leq m$.

If there is a pancyclic arc in the path $\left(v_{0}, v_{1}, \ldots, v_{k-1}\right)$, the cycle $C$ contains five pancyclic arcs. So, by Lemma 9, we may assume that $N_{T_{1}}^{+}\left(v_{k-1}\right) \subseteq N_{T_{1}}^{+}\left(v_{k-2}\right) \subseteq \ldots \subseteq N_{T_{1}}^{+}\left(v_{0}\right)$. There are two distinct vertices $r_{1}$ and $r_{2}$ of $T_{1}$ that are dominated by $v_{k-1}$. Let $C_{1}$ be a hamiltonian cycle of $T_{1}$ containing three $T_{1}$-pancyclics arcs. Let $r_{1}^{-}$(resp. $r_{2}^{+}$) be the vertex dominating $r_{1}$ (resp. $\left.r_{2}\right)$ in $C$. Let $P_{1}$ (resp. $P_{2}$ ) be the subpaths of $C_{1}$ with beginning $r_{1}$ (resp. $r_{2}$ ) and end $r_{2}^{-}$(resp. $r_{1}^{-}$).

1) Suppose first that $m \geq 3$.

Let $C^{\prime}$ be the cycle defined as follows:

- If $k \geq 3$, then $C^{\prime}:=\left(v_{0}, v_{k-1}, P_{1}, t_{m}, v_{1}, v_{2}, \ldots, v_{k-2}, P_{2}, t_{2}, t_{3}, \ldots, t_{m-1}, v_{0}\right)$;

- if $k=2$, then $C^{\prime}:=\left(v_{0}, P_{2}, t_{m}, v_{1}, P_{1}, t_{2}, t_{2}, t_{3}, \ldots, t_{m-1}, v_{0}\right)$.

INRIA 
Since $C_{1}$ contains at least three $T_{1}$-pancyclic arcs, one of them, say $e$ is contained in $P_{1}$ or $P_{2}$. So, by Lemma $6, e$ is pancyclic in $T$. Moreover $C^{\prime}$ contains the four arcs $\left(t_{m}, v_{1}\right),\left(t_{m-1}, v_{0}\right),\left(v_{k-2}, r_{2}\right)$ and $\left(v_{k-1}, r_{1}\right)$. By Lemma 1 , these arcs are pancyclic in respectively in $T-\left[v_{0}\right], T-\left[t_{m}\right], T-\left[v_{k-1}\right]$ and $T$. Hence because they are contained in $C^{\prime}$, these four arcs are pancyclic. Thus $C^{\prime}$ contains five pancyclic arcs.

2) Suppose now that $m=2$. Then $v_{0}$ (and then every $v_{j}, 0 \leq j \leq k-1$ ) has an inneighbour in $T_{1}$ which dominates $X$. Without loss of generality we may suppose that this inneighbour $s_{2}^{-}$is between $r_{1}$ and $r_{2}$ in $C_{1}$, that $s_{2}$ the successor of $s_{2}^{-}$along $C_{1}$ dominates $v_{0}$. Let $Q_{1}$ (resp. $Q_{2}$ ) be the path beginning in $r_{1}$ (resp. $s_{2}$ ) and ending in $s_{2}^{-}$(resp. $\left.r_{1}^{-}\right)$.

Set $C_{0}:=\left(v_{0}, Q_{2}, t_{2}, v_{1}, v_{2}, \ldots, v_{k-1}, Q_{1}, v_{0}\right)$. It is easy to check that $\left(v_{0}, s_{2}\right)$ and $\left(t_{2}, v_{1}\right)$ and $\left(v_{k-1}, r_{1}\right)$ are pancyclic in $T$. If $P_{1} \cup P_{2}$ contains two $T_{1}$-pancyclic arcs then these two are also pancyclic in $T$ by Lemma 6 . So $C_{0}$ contains five pancyclic arcs.

Hence we may assume that both $\left(r_{1}^{-}, r_{1}\right)$ and $\left(s_{2}^{-}, s_{2}\right)$ are pancyclic. Let $e$ be the third $T_{1}$-pancyclic arc. Let $x$ be an vertex of $Q_{1}$ such that $v_{o} \rightarrow x$ and its successor $x^{+}$in $Q_{1}$ dominates $v_{0}$. Such a vertex exists since $v_{0} \rightarrow r_{1}$ and $s_{2}^{-} \rightarrow v_{0}$. The arc $\left(x, x^{+}\right)$of $Q_{1}$ is pancyclic. Indeed ...

Thus if $e=\left(x, x^{+}\right)$, so $e \in Q_{1}$.

Three cases may arise:

- a) Suppose that $w=s_{2}=r_{1}^{-}$. Then $T_{1}-\left[s_{2}^{-}, s_{2}\right] \rightarrow s_{2}^{-}$, thus $\left(s_{2}^{-}, v_{0}, r_{1}, s_{2}^{-}\right)$is a 3-cycle. For $4 \leq l \leq n_{1}+1,\left(s_{2}^{-}, v_{0}\right)$ is contained in the $l$-cycle obtained by replacing the arc $\left(s_{2}^{-}, s_{2}\right)$ by $\left(s_{2}^{-}, v_{0}, s_{2}\right)$ in the $l-1$-cycle. Now since $v_{0} \rightarrow X-v_{0}$ and $X \rightarrow r_{1}$, by Proposition 3 (applied for every vertex of $X-v_{0}$ one after another), $\left(s_{2}^{-}, v_{0}\right)$ is contained in an $l$-cycle for $n_{1}+2 \leq l \leq n-1$. And it is contained in $C_{0}$. Hence, $\left(s_{2}^{-}, v_{0}\right)$ is pancyclic and $C_{0}$ contains five pancyclic arcs.

- b) Suppose that $w=r_{1}$. Then $r_{1} \rightarrow T_{1}-Q_{2}$ and $Q_{2} \rightarrow r_{1}$. Then $\left(s_{2}^{-}, v_{0}, r_{1}, s_{2}^{-}\right)$ is a 3-cycle. For $4 \leq l \leq n_{1}+1$, pick $Q_{1}^{\prime}$ a subpath of $Q_{1}$ ending in $s_{2}^{-}$of length $l_{1}<\left|Q_{1}\right|-2$ and $Q_{2}^{\prime}$ a subpath of $Q_{2}$ beginning in $s_{2}$ of length $l_{2}<\left|Q_{2}\right|-2$ such that $l_{1}+l_{2}+4=l$, then $\left(s_{2}^{-}, v_{0}, Q_{2}^{\prime}, r_{1}, Q_{2}^{\prime}\right)$ is an $l$-cycle. Analogously to the end of Case a), we obtain that $\left(s_{2}^{-}, v_{0}\right)$ is pancyclic and $C_{0}$ contains five pancyclic arcs.

- Suppose that $w=s_{2}^{-}=x^{+}$. Then $r_{1} \rightarrow w$, so $\left(s_{2}^{-}, v_{0}, r_{1}, s_{2}^{-}\right)$is a 3 -cycle. And $T_{1}-s_{2}^{-}$is a transitive tournament with outgenerator $s_{2}$ and ingenerator $x$. Thus for $4 \leq l \leq n_{1}+1$, in $T_{1}-s_{2}^{-}$, there is an $\left(s_{2}, x\right)$-path $P$ of lenght $l-2$. Hence, $\left(Q, s_{2}^{-}, v_{0}, s_{2}\right)$ is an $l$-cycle. Again, analogously to the end of Case a), we obtain that $\left(s_{2}^{-}, v_{0}\right)$ is pancyclic and $C_{0}$ contains five pancyclic arcs.

III) Suppose now that $T-X$ is a transitive tournament. 
If $v_{0} \rightarrow t_{n-k-1}$, then $X^{\prime}=T\left[t_{n-k}, v_{1}, v_{2}, \ldots, v_{k-1}\right]$ is a reductor of $T$ and $T-X^{\prime}$ is not a transitive. Indeed $v_{0}$ is dominated by some vertex $t_{i}$ and then $\left(t_{i}, v_{o}, t_{1}, t_{i}\right)$ is a 3 -cycle. So we have the result by one of the previous case.

Analosgously, we obtain the result if $v_{k-2} \leftarrow t_{2}$. Thus we may assume that $v_{0} \leftarrow t_{n-k-1}$ and $v_{k-2} \rightarrow t_{2}$.

By Lemma $1,\left(t_{n-k-1}, v_{0}\right)$ is pancyclic in $T-t_{n-k}$ and $\left(t_{n-k}, v_{1}\right)$ is pancyclic in $T-v_{0}$. Thus two arcs are $T$-pancyclic because they are contained in the $n$-cycle $C_{3}=\left(t_{n-k-1}, v_{0}, v_{k-1}, t_{2}, t_{3}, \ldots, t_{n-k-2}, t_{n-k}, v\right.$ if $k \geq 3$ or $C_{2}=\left(t_{n-3}, v_{0}, t_{1}, t_{n-2}, v_{1}, t_{2}, t_{3}, \ldots, t_{n-3}\right)$ if $k=2$. Analogously, $\left(v_{k-1}, t_{2}\right)$ and $\left(v_{k-2}, t_{1}\right)$ are also $T$-pancyclic.

Suppose that $k \geq 3$, then the 4 pancyclic $\operatorname{arcs}\left(t_{n-k-1}, v_{0}\right),\left(t_{n-k}, v_{1}\right),\left(v_{k-1}, t_{2}\right)$ and $\left(v_{k-2}, t_{1}\right)$ are contained in the two $n$-cycles $C_{3}$ and $C_{3}^{\prime}=\left(t_{n-k-1}, v_{0}, v_{k-1}, t_{2}, t_{n-k}, v_{1}, v_{2}, \ldots, v_{k-2}, t_{1}, t_{3}, t_{4}, \ldots, t_{n-k-1}\right)$. Therefore no arcs in $\left\{\left(t_{i}, t_{i+1}\right), 2 \leq i \leq n-k-2\right\}$ is $T$ pancyclic. By Lemma $3, t_{i} \rightarrow v_{0}$ for $2 \leq i \leq n-k$ and $\left(t_{1}, t_{2}\right)$ is pancyclic, and $v_{k-1} \rightarrow t_{i}$ for $1 \leq i \leq n-k-1$ and $\left(t_{n-k-1}, t_{n-k}\right)$ is pancyclic. Thus at least one of the $\operatorname{arcs}\left(v_{i}, v_{i+1}\right)$ is in a 3 -cycle is pancyclic by Lemma 9 . Hence the cycle $C=\left(v_{0}, v_{1}, \ldots, v_{k-1}, t_{1}, t_{2}, \ldots, t_{n-k}, v_{0}\right)$ contains five pancyclic arcs.

Thus we may assume that $k=2$. We may assume that there is no pancyclic arcs in $\left\{\left(t_{i}, t_{i+1}\right), 2 \leq i \leq n-4\right\}$, otherwise $C_{2}$ contains five pancyclic arcs. Then by Lemma 3 , $t_{i} \rightarrow v_{0}$ for $2 \leq i \leq n-2$ and $\left(t_{1}, t_{2}\right)$ is pancyclic, and $v_{1} \rightarrow t_{i}$ for $1 \leq i \leq n-3$ and $\left(t_{n-3}, t_{2}\right)$ is pancyclic. Hence $\left(v_{0}, v_{1}\right)$ is in the 3 -cycle $\left(v_{0}, v_{1}, t_{2}, v_{0}\right)$, so by Lemma 9 , it is pancyclic. Hence the cycle $C=\left(v_{0}, v_{1}, t_{1}, t_{2}, \ldots, t_{n-2}, v_{0}\right)$ contains five pancyclic arcs.

This result is best possible since the regular tournament $R_{5}$ on five vertices is 2 -strong and obviously satisfies $h\left(R_{5}\right)=5$.

It follows directly from Theorems 5 and Equation 6 , that for $k \geq 2$, every $k$-strong tournament has at least $2 k+2$ pancyclics arcs.

Corollary 3 Every $k$-strong tournament has at least $2 k+2$ pancyclic arcs.

We now prove a slighty better result.

Theorem 6 Every $k$-strong $(k \geq 2)$ tournament has at least $2 k+3$ pancyclic arcs.

Proof. Let $x$ and $y$ be vertices such that the arcs of $A^{+}(x) \cup A^{-}(y)$ are pancyclic. By Equation 7, we have the result, if $d^{+}(x) \geq k+1$ or $d^{-}(y) \geq k+1$ or $A^{+}(x) \cap A^{-}(y)=\emptyset$. Thus we may assume that $x \rightarrow y$, and $d^{+}(x)=d^{-}(y)=k$.

Then $X=N^{+}(x)$ is a reductor of $T$ containing $y$. We have $2 k-1$ pancyclic arcs in $A^{+}(x) \cup A^{-}(y)$ and by Corollary 1 , there is at least one pancyclic $\operatorname{arc} e_{1}$ in $A(X, O u t(T-X))$.

If $X$ is not transitive, then according to Lemma 2, there are at least three pancyclic arcs in $X$, with at most one of them in $A^{-}(y)$. And by Proposition 8, there is at most one pancyclic arc in $T-X$. It follows that $p(t) \geq 2 k+3$. Hence we may assume that $X$ is the transitive tournament $T T\left(v_{0}, v_{1}, \ldots, v_{k-1}\right)$.

Let $T_{1} \rightarrow T_{2} \rightarrow \cdots \rightarrow T_{m}$ be a decomposition of $T-X$. By Lemmas 4,5 and 6 , at most one of the $T_{i}$ is not reduced to the vertex $t_{i}$. 
Suppose that for some $2 \leq i \leq m-1, T_{i}$ is not reduced to a single vertex. If $\left|T_{i}\right| \geq 4$ then by Lemma 4 , there are at least three pancyclic arcs in $T_{i}$. Thus $p(t) \geq 2 k+3$. Asssume now that $T_{i}$ is a 3 -cycle. Two arcs of $T_{i}$ are pancyclic by Lemma 5 . Let $t_{i}$ be a vertex of $T_{i}$. By Lemma 10, if $t_{i} \rightarrow v_{0}$, then $\left(t_{i-1}, t_{i}\right)$ is pancyclic and if $t_{i} \leftarrow v_{0}$, then $\left(t_{i}, t_{i+1}\right)$ is pancyclic. Hence, $p(T) \geq 2 k+3$.

Suppose now that $T_{1}$ is not reduced to a single vertex. By Lemma 7 , we have the result if $h\left(T_{1}\right) \geq 4$. So we may assume that $T_{1} \in \mathcal{P}_{3}$. Also by Lemma 7 , there are two pancyclic arcs in $T_{1}$. According to Proposition 7 (dual), there are two distinct vertices $w_{1}$ and $w_{2}$ of $T_{1}$ such that $v_{k-1} \rightarrow w_{1}$ and $v_{k-2} \rightarrow w_{2}$. Let $C_{1}$ be a hamiltonian cycle of $T_{1}$.

- Suppose first that $m \geq 3$. If a vertex $t_{i}$ with $2 \leq i \leq m-1$ dominates an element of $X$ then by Lemma 10 one of the arcs $\left(t_{j}, t_{j+1}\right)$ with $i \leq j \leq m-1$ is pancyclic. Hence $p(T) \geq 2 k+3$. So we may assume that $T-\left[T_{1}, X\right] \rightarrow X$. Let us prove that $\left(v_{k-2}, w_{2}\right)$ is pancyclic : it is contained in the hamiltonian cycle $\left(P_{2}, t_{m}, v_{k-1}, P_{1}, t_{2}, t_{3}, \ldots, t_{m-1}, v_{0}, v_{1}, \ldots v_{k-2}, w_{2}\right)$, where $P_{1}$ (resp. $\left.P_{2}\right)$ is the subpath along $C_{1}$ of $T_{1}$ starting in $w_{1}$ (resp. $w_{2}$ ) and ending in the predecessor of $w_{2}$ (resp. $w_{1}$ ). For $3 \leq l \leq n-1$, let $0 \leq k^{\prime} \leq k-2,0 \leq m^{\prime} \leq m-2$ and $0 \leq l_{1} \leq n_{1}-1$ such that $l_{1}+k^{\prime}+m^{\prime}+3=l$. There is a path $Q_{1}$ of length $l_{1}$ starting in $w_{2}$. Thus $\left(v_{k-2}, w_{2}\right)$ is contained in the $l$-cycle $\left(v_{k-2}, Q_{2}, t_{m-m^{\prime}}, t_{m-m^{\prime}+1}, \ldots, t_{m}, v_{k-2}-k^{\prime}, v_{k-1-k^{\prime}}, \ldots, v_{k-2}\right)$.

Hence $T$ has at least $2 k+3$ pancyclic arcs.

- Suppose now that $m=2$. Since $d_{X}^{-}\left(v_{0}\right) \leq k-2, v_{0}$ is dominated by at least one vertex in $T_{1}$. Hence there exists a vertex $w_{0}$ of $T_{1}$ dominated by $v_{0}$ such that its predecessor $w_{0}^{-}$along $C_{1}$ dominates $v_{0}$.

Because $d_{X}^{+}\left(v_{k-1}\right) \leq k-2, v_{k-1}$ dominates at least two vertices of $T_{1}$, so at least one, say $w_{1}$, distinct of $w_{0}$.

Let us now prove that $\left(v_{0}, w_{0}\right)$ is pancyclic. For $3 \leq l \leq n_{1}+1$, let $Q_{0}$ be a path in $T_{1}$ starting at $w_{0}$ of length $l-3$. Then $\left(v_{0}, Q_{0}, t_{2}, v_{0}\right)$ is an $l$-path. Let $Q_{1}$ (resp. $\left.Q_{2}\right)$ be the subpath of $C_{1}$ starting at $w_{1}$ (resp. $w_{0}$ ) and ending in $w_{0}^{-}$(resp. the predecessor of $\left.w_{1}\right)$ along $C_{1}$. Then for $n_{1}+2 \leq l \leq n,\left(Q_{1}, v_{0}, Q_{2}, t_{2}, v_{n+1-l}, v_{n-l}, \ldots, v_{k-1}, w_{1}\right)$ is an $l$-path containing $\left(v_{0}, w_{0}\right)$.

Hence $T$ has at least $2 k+3$ pancyclic arcs.

Suppose now that $T-X$ is the transitive tournament $T T\left(t_{1}, t_{2}, \ldots, t_{n-k}\right)$.

Let $i_{0}$ be the smallest integer $i>1$ such that $v_{k-1} \rightarrow t_{i}$.

- Suppose that $i_{0}=2$. Since $T$ is 2 -strong, $v_{0}$ is dominated by vertex $t_{i_{1}}$ disticnt from $t_{n-k}$. By Lemma $1,\left(v_{k-1}, t_{2}\right)$ and $\left(v_{k-2}, t_{1}\right)$ are respectively $\left(T-t_{1}\right)$ - and $\left(T-v_{k-1}\right)$ pancyclic, thus they are contained in $l$-cycle for any $3 \leq l \leq n-1$. And they both are in the following hamiltonian cycle : $\left(v_{k-1}, t_{2}, t_{3}, \ldots, t_{i_{1}}, v_{0}, v_{1}, \ldots, v_{k-2}, t_{1}, t_{i_{1}+1}, t_{i_{1}+2}, \ldots t_{n-k}, v_{k-1}\right)$. Thus they both are pancyclic in $T$. Moreover, by Proposition 8 , there is a $T$-pancyclic arc in $T-X$. Hence, $p(T) \geq 2 k+3$. 
- Suppose now that $i_{0}>2$. Then $X^{\prime}=T\left[X-v_{-1}, t_{1}\right]$ is a reductor of $X$. And by Lemma $3,\left(t_{1}, t_{2}\right)$ is $T$-pancyclic.

The subtournament $T^{\prime}=T\left[t_{i_{0}}, t_{i_{0}+1}, \ldots t_{n-k}, v_{k-1}\right]$ is a strong component of $T-X^{\prime}$. By Lemma 7, there are $h\left(T^{\prime}\right)-1 T$-pancyclic arcs in $T^{\prime}$. And at most one of them is in $A^{+}(x)$. Thus, if $h\left(T^{\prime}\right) \geq 4$, we obtain $p(T) \geq 2 k+3$. So we may assume that $h\left(T^{\prime}\right)=3$, so $T^{\prime} \in \mathcal{P}_{3}$. Since $T^{\prime}-v_{k-1}$ is transitive, $\left(t_{n-k}, v_{k-1}\right)$ and $\left(v_{k-1}, t_{i_{0}}\right)$ are $T^{\prime}$-pancyclic. Let $e_{2}$ be the third $T^{\prime}$-pancyclic arc. By Lemma $6, e_{2}$ is also $T$-pancyclic because $t_{n-k}=x$ dominates $v_{0}$.

If $v_{0}$ is dominated by a vertex of $T^{\prime}-\left[x, v_{k-1}\right]$ then by Lemma $6,\left(v_{k-1}, t_{i_{0}}\right)$ is pancyclic in $T$ and so $p(T) \geq 2 k+3$.

If $v_{0}$ dominates $T^{\prime}-\left[x, v_{k-1}\right]$, it must have an ineighbour $t_{i_{2}}$ with $2 \leq i_{2} \leq i_{0}-1$. Hence, by Proposition 10, there is a pancyclic arc $\left(t_{i}, t_{i+1}\right)$ with $1 \leq i \leq i_{0}-2$. So, $p(T) \geq 2 k+3$.

\section{$5 \quad$ Tournaments with exactly four pancyclic arcs}

We now prove a generalization of Lemma 3 .

Lemma 10 Let $T$ be a strong tournament, $X$ a reductor of $T$ and $T_{1} \rightarrow T_{2} \rightarrow \cdots \rightarrow T_{m}$ be a decomposition of $T-X$.

i) For $1<i<m-1$, if there exist $t_{i} \in T_{i}$ and $t_{i+1} \in T_{i+1}$ such that $\left(t_{i}, t_{i+1}\right)$ is in a 3 -cycle then $\left(t_{i}, t_{i+1}\right)$ is pancyclic.

ii) If $m \geq 3$ and $T_{1}=\left\{t_{1}\right\}$ and there is a vertex $t_{2} \in T_{2}$ such that $\left(t_{1}, t_{2}\right)$ is in a 3 -cycle then $\left(t_{1}, t_{2}\right)$ is pancyclic.

Proof. Let $\left(v_{0}, v_{1}, \ldots, v_{k-1}\right)$ be a hamiltonian path of $X$ and for $1 \leq i \leq m$, set $n_{i}=\left|T_{i}\right|$.

i) Let $v$ be the vertex of $X$ such that $\left(t_{i}, t_{i+1}, v, t_{i}\right)$ is a 3-cycle. Then $v$ belongs to $X$ and then by Proposition 6, dominates a vertex $t \in T_{1}$. Then $\left(t_{i}, t_{i+1}, v, t, t_{i}\right)$ is a 4 -cycle. Let $t_{1}$ be an element of $T_{1}$ that is dominated by $v_{k-1}$ and $t_{m}$ be an element of $T_{m}$ dominating $v_{0}$. Let $5 \leq l \leq n$. The arc $\left(t_{i}, t_{i+1}\right)$ is contained in a cycle of length $l$. Indeed, take $0 \leq k^{\prime}<k$, $0<l_{1}<\sum_{j=1}^{i} n_{j}$ and $0 \leq l_{2}<\sum_{j=i+1}^{m} n_{j}$ such that $k^{\prime}+l_{1}+l_{2}+3=l$. By Proposition 5 , there is a $\left(t_{1}, t_{i}\right)$-path $P_{1}$ of length $l_{1}$ in $T\left[T_{1}, \ldots, T_{i}\right]$, and a $\left(t_{i+1}, t_{m}\right)$-path $P_{2}$ of length $l_{2}$ in $T\left[T_{i+1}, \ldots, T_{m}\right]$. Then $\left(t_{i}, P_{2}, v_{0}, v_{1}, \ldots, v_{k^{\prime}}, P_{1}\right)$ is an $l$-cycle containing $\left(t_{i}, t_{i+1}\right)$. Hence $\left(t_{i}, t_{i+1}\right)$ is pancyclic.

ii) Let $4 \leq l \leq n$. Let us prove that $\left(t_{1}, t_{2}\right)$ is contained in an $l$-cycle. By Proposition 6 , $v_{k-1} \rightarrow t_{1}$. Let $t_{m}$ be an element of $T_{m}$ dominating $v_{0}$. Since $m \geq 3, T-\left[X, t_{1}\right]$ is reducible and $t_{2}$ is one of its outgenerators and $t_{m}$ one of its ingenerators. Therefore, by Proposition 5 , there is a $\left(t_{1}, t_{m}\right)$-path $P$ of length $l-3$ in $T-\left[X, t_{1}\right]$. Then $\left(P, v_{0}, v_{1}, \ldots, v_{k-1}, t_{1}, t_{2}\right)$ is the desired $l$-cycle.

INRIA 
Definition 2 A tournament is in $\mathcal{P} 4$ if there is a vertex $v$ such that $T-v$ is the transitive tournament $T T\left[t_{1}, t_{2}, \ldots, t_{m}\right]$ and three integers $1<i_{1}<i_{2}<i_{3} \leq m$ such that $v \rightarrow t_{j}$ if and only if $1 \leq j<i_{1}$ or $i_{2} \leq j<i_{3}$.

Theorem 7 A tournament has exactly 4 pancyclic arcs if and only if it is in $\mathcal{P}_{4}$.

Proof. It is easy to check that every tournament of $\mathcal{P}_{4}$ has exactly four pancyclic arcs.

Let $T$ be a tournament with exactly 4 pancyclic arcs. By Corollary $3, T$ is $(=1)$-strong. Let $\{v\}$ be a reductor of $X$ and $T_{1} \rightarrow T_{2} \rightarrow \cdots \rightarrow T_{m}$ be a decomposition of $T-v$. By Equation 2, at most one of the $T-i, 1 \leq i \leq m$ is not reduced to a single vertex.

Let us prove that there exists a decomposition such that each $T_{i}$ is reduced to a single vertex.

1. If $T-v$ is a transitive tournament, then by Lemma $3, T$ is in $\mathcal{P}_{4}$.

2. Suppose that there exist $1<i<m$, such that $T_{m}$ is not reduced to a vertex. By Corollary $1,\left(t_{m}, v\right)$ and $\left(v, t_{1}\right)$ are pancyclic. By Equation 2 , then $\left|T_{i}\right|=3$. If $T_{i} \rightarrow v$ or $v \rightarrow T_{i}$ then the three $\operatorname{arcs}$ of $T_{i}$ are pancyclic, then $T$ has 5 pancyclic arcs. This is a contradiction. Then there is a vertex $t_{i} \in T_{i}$ such that $t_{i} \rightarrow v$. Thus there is an arc $\left(t_{j}, t_{j+1}\right)$ with $1 \leq j<i$ such that $\left(v, t_{j}, t_{j+1}, v\right)$ is a 3 -cycle. So by Lemma 10 , $\left(t_{j}, t_{j+1}\right)$ is pancyclic. Again $T$ has 5 pancyclic arcs which is a contradiction.

3. Suppose that $T_{m}$ is not reduced to a vertex. By Equation 2, $v$ has a unique inneighbour $u$ in $T_{m}$. Set $T_{i}=\left\{t_{i}\right\}$ for $1 \leq i \leq m-1$. By Corollary $1,(u, v)$ and $\left(v, t_{1}\right)$ are pancyclic and by Lemma 6 dual, there are two pancyclics arcs in $T_{m}$. Therefore, there is no other pancyclic arcs in $T$. Thus according to Lemma $10, v \rightarrow\left\{t_{i}, 1 \leq i \leq m-1\right\}$. Then $u$ is a reductor. And there are 3 pancyclic arcs which are not in $T_{m}-u$. Then by Lemmas 4,5 and $7, T-u$ is a transitive tournament. So we have the result by Case 1 .

\section{Tournaments with exactly five pancyclic arcs}

Definition 3 A tournament is in $\mathcal{P} 5$ if there is a vertex $v$ such that $T-v$ is the transitive tournament $T T\left[t_{1}, t_{2}, \ldots, t_{m}\right]$ and three integers $1<i_{1}<i_{2}<i_{3}<i_{4}<i_{5} \leq m$ such that $v \rightarrow t_{j}$ if and only if $1 \leq j<i_{1}$ or $i_{2} \leq j<i_{3}$ or $i_{4} \leq j<i_{5}$.

The tournament $Q(n)(n \geq 5)$ is the tournament contructed from the transitive tournament $T T_{n-4}=T T\left(t_{1}, t_{2}, \ldots t_{n-4}\right)$ and four vertices $a, b, c$ and $v$ such that $(a, b, c, a)$ is a 3-cycle, $v \rightarrow T T_{n-4}, T T_{n-4} \rightarrow\{a, b, c\},\{a, b\} \rightarrow v$ and $c \leftarrow v$.

\section{Proposition 9}

$$
p(Q(5))=5
$$

For $n \geq 6, p(Q(n))=6$. 
Proof. $v$ is a reductor of $T$. By Corollary $1,(a, v),(b, v)$ and $\left(v, t_{1}\right)$ are pancyclic. And By Lemma $6,(a, b)$ and $(c, a)$ are pancyclic. Thus $p(Q(n)) \geq 5$.

Now an arc in $T T_{n-4}$ is not pancyclic in $T$ because it is contained in no 3-cycle; $(b, c)$ is not pancyclic because it is contained in no 4-cycle ; and $(v, c)$ is not pancyclic because it is contained in no $l$-cycle for $l \geq 5$. For $1 \leq i<n-4$ the arc $\left(t_{i}, x\right)$ with $x \in\{a, b, c\}$ is not pancyclic because it is contained in no hamiltonian cycle. The arc $\left(t_{n-4}, a\right)$ is not pancyclic since it is contained in no hamiltonian cycle and $\left(t_{n-4}, c\right)$ is not pancyclic since it is contained in no 3 -cycle

For $3 \leq l \leq n-2$, the arc $\left(t_{n-4}, b\right)$ is contained in the $l$-cycle $\left(t_{n-4}, b, v, t_{n-1-l}, \ldots, t_{n-4}\right)$. For $5 \leq l \leq n$, the arc $\left(t_{n-4}, b\right)$ is contained in the $l$-cycle $\left(t_{n-4}, b, c, a, v, t_{n+1-l}, \ldots, t_{n-4}\right)$. Thus, if $n \geq 6, p(Q(n))=6$. It is easy to check that if $n=5$, the arc $\left(t_{1}, b\right)$ is contained in no 4-cycle. Thus $p(Q(5))=5$.

Theorem 8 A tournament has exactly 5 pancyclic arcs if and only if it is in $\mathcal{P}_{5}$ or is $Q(5)$.

Proof. It is easy to check that every tournament of $\mathcal{P}_{5} \cup\{Q(5)\}$ has exactly five pancyclic arcs.

Let $T$ be a tournament with exactly 5 pancyclic arcs. By Corollary $3, T$ is $(=1)$-strong. By Equation 2, at most one of the $T_{i}, 1 \leq i \leq m$ is not reduced to a single vertex.

- If $T-v$ is a transitive tournament. Then by Lemma $3, T$ is in $\mathcal{P}_{5}$.

- Suppose that there exist $1<i<m$, such that $T_{m}$ is not reduced to a vertex. By Corollary $1,\left(t_{m}, v\right)$ and $\left(v, t_{1}\right)$ are pancyclic. By directionnal duality, we may suppose that there is a vertex $t_{i} \in T_{i}$ such that $t_{i} \rightarrow v$. Then by Lemma 10, there is a pancyclic arc in $T\left[t_{1}, t_{2}, \ldots, t_{i}\right]$. Thus, there are at most two pancyclic $\operatorname{arc}$ in $T_{i}$. Then by Lemma 4 and the proof of Lemma $5, T_{i}$ is a 3-cycle containing 2 pancyclic arcs and there is a vertex $s_{i} \in T_{i}$ dominated by $v$. Then by Lemma 10, there is a pancyclic arc in $T\left[s_{i}, t_{i+1}, \ldots, t_{m}\right]$. So $p(T) \geq 6$ which is a contradiction.

- Suppose that $T_{m}$ is not reduced to a vertex.

Then $\delta^{+} \geq 2$. So by Equation $5, h(T) \leq 4$ thus by Theorem $1, T_{m} \in \mathcal{P}_{3}$. Let $w$ be a reductor of $T_{m}$ such that $T_{m}-w$ is the transitive tournament $T T\left[a_{1}, \ldots, a_{p}\right]$. Let $i$ be the index such that $w \rightarrow a_{i}$ and $w \leftarrow a_{i+1}$.

$v \rightarrow T_{m} \backslash\left\{a_{i}, w, a_{p}\right\}$, otherwise by Lemma $6, h(T) \geq 5$ which is a contradiction. Also $v \rightarrow T-\left[v, T_{m}\right]$ oterwise by Lemma 10 , there is a pancyclic arc in $\left\{\left(t_{j}, t_{j+1}\right), 1 \leq j \leq\right.$ $m-2\}$.

By Equation $2, h_{T_{m}}^{-}(v) \leq 2$.

If $h_{T_{m}}^{-}(v)=1$, then let $u$ be the inneighbour of $v$ in $T_{m}$. The vertex $u$ is a reductor. And $\operatorname{out}(T-u)<\operatorname{out}(T-v)$. Iterating the process, we find a reductor $v^{\prime}$ such that $T-v^{\prime}$ is transisitve or $h_{T_{m}}^{-}\left(v^{\prime}\right)=2$. So we may assume that $h_{T_{m}}^{-}(v)=2$. 
- Suppose that $N_{T_{m}}^{-}(v)=\left\{a_{i}, w\right\}$. If $i \neq 1$, then by Lemma 6 dual, the three $\operatorname{arcs}$ $\left(w, a_{1}\right),\left(a_{p}, w\right)$ and $\left(a_{i}, a_{i+1}\right)$ are pancyclic. So $p(T) \geq 6$ which is a contradiction. Thus $i=1$. It is easy to see that $\left(a_{1}, a_{2}\right)$ is contained in every cycle of length $l$, for $5 \leq l \leq n$. It follows that $\left|T_{m}\right|=3$ Then $T=Q(n)$ and by Proposition 9 , $T=Q(5)$.

- If $N_{T_{m}}^{-}(v)=\left\{w, a_{p}\right\}$, then by Lemma 6 dual, $\left(a_{p}, w\right)$ and $\left(a_{i}, a_{i+1}\right)$ are pancyclic. It is easy to see that $\left(w, a_{1}\right)$ is contained in every cycle of length $l$, for $5 \leq l \leq n$. And because $\left(w, a_{1}\right)$ is pancyclic in $T_{m}$, it follows that $\left|T_{m}\right|=3$. Thus $T=Q(n)$ and by Proposition 9, $T=Q(5)$.

- Suppose that $N_{T_{m}}^{-}(v)=\left\{a_{i}, a_{p}\right\}$. If $i \neq p-1$, then by Lemma 6 dual, the three $\operatorname{arcs}\left(w, a_{1}\right),\left(a_{p}, w\right)$ and $\left(a_{i}, a_{i+1}\right)$ are pancyclic. So $p(T) \geq 6$ which is a contradiction. Thus $i=p-1$. It is easy to see that $\left(a_{p}, w\right)$ is contained in every cycle of length $l$, for $5 \leq l \leq n$. And because $\left(a_{p}, w\right)$ is pancyclic in $T_{m}$, it follows that $\left|T_{m}\right|=3$. So $T=Q(n)$ and by Proposition $9, T=Q(5)$.

\section{References}

[1] B. Alspach, Cycles of each length in regular tournaments, Canad. Math. Bull. 10 (1967), $283-286$.

[2] P. Camion, Chemins et circuits hamiltoniens des graphes complets, C.R. Acad. Sci. Paris 249 (1959), 2151-2152. , J. Combin. Inform. System Sci., 19 (3-4)(1994), 207214.

[3] J. W. Moon, Topics on Tournaments, (Holt, Rinehart and Winston, New York) (1968).

[4] J. W. Moon, On $k$-cyclic and pancyclic arcs in strong tournaments, J. Combin. Inform. System Sci., 19 (3-4)(1994), 207-214.

[5] T. Yao, Y. Guo and K. Zhang, Pancyclic out-arcs of a vertex in tournaments, Discrete Appl. Math., 99 (1-3)(2000), 245-249. 


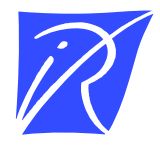

Unité de recherche INRIA Sophia Antipolis 2004, route des Lucioles - BP 93 - 06902 Sophia Antipolis Cedex (France)

Unité de recherche INRIA Lorraine : LORIA, Technopôle de Nancy-Brabois - Campus scientifique 615, rue du Jardin Botanique - BP 101 - 54602 Villers-lès-Nancy Cedex (France)

Unité de recherche INRIA Rennes : IRISA, Campus universitaire de Beaulieu - 35042 Rennes Cedex (France)

Unité de recherche INRIA Rhône-Alpes : 655, avenue de l'Europe - 38330 Montbonnot-St-Martin (France)

Unité de recherche INRIA Rocquencourt : Domaine de Voluceau - Rocquencourt - BP 105 - 78153 Le Chesnay Cedex (France)

Éditeur

INRIA - Domaine de Voluceau - Rocquencourt, BP 105 - 78153 Le Chesnay Cedex (France)

http://www.inria.fr

ISSN 0249-6399 